\title{
Development of non-conservative joints in beam networks for vibration energy flow analysis
}

\author{
Jee-Hun Song and Suk-Yoon Hong* \\ Department of Naval Architecture and Ocean Engineering, Seoul National University, \#56-1, Shinlim-dong, \\ Kwanak-gu, Seoul 151-742, Korea
}

Received 30 March 2005

\begin{abstract}
Our work aims to find a general solution for the vibrational energy flow through a plane network of beams on the basis of an energy flow analysis. A joint between two semi-infinite beams are modeled by three sets of springs and dashpots. Thus, the results can incorporate the case of complaint and non-conservative in all the three degrees of freedom. In the cases of finite coupled structures connected at a certain angle, the derived non-conservative joints and developed wave energy equation were applied. The joint properties, the frequency, the coupling angle, and the internal loss factor were changed to evaluate the proposed methods for predicting medium-to-high frequency vibrational energy and intensity distributions.
\end{abstract}

\section{Introduction}

Many engineering structures are made up from elements, such as rods, beams, membranes and plates, which are joined together either by rivets, bolts or welds. At low frequency ranges, the vibrational response of these structures may be analyzed with regards to structural deformation by conventional methods such as finite element method (FEM) and boundary element method (BEM). However, as the frequency is increased, the methods come to need higher order shape function or large number of fine elements, and their results become inaccurate. Thus, the FEM and BEM are very expensive and not effective in high frequency range. In recent decades, the statistical energy analysis (SEA) has been developed for high frequency analysis, which provides one averaged value of energy density in a subsystem. From SEA, any detail information such as variation of energy density and energy flow paths in a subsystem cannot be obtained, and the size of subsystem in a structure should be larger when the frequency of interest is decreased. To improve the weakness of SEA in high frequency range and to overcome the limit of frequency of the FEM and BEM, some alternative methods have been investigated by many researchers. Energy flow analysis (EFA) method, one of the alternative methods, has been introduced by Belov et al. [1]. EFA method is based on an energy governing equation analogous to the heat conductivity equation, of which primary variable is energy density. Using this method at high frequencies, the spatial variation of the time- and locally space-averaged vibration energy density and energy transmission paths in a structure can be effectively predicted. Moreover, EFA method has been considered appropriate to the vibration analysis in the middle frequency range in which the FEM, BEM and SEA are not proper to be applied. Nefske and Sung [2] applied the energy flow finite element method (EFFEM) for predicting the flexural vibration of beams. Wohlever and Bernhard [3-6] extended this method to energy flows in rods, beams, membranes and thin plates. Cho [7] used EFA to predict the vibration response of the coupled structures by applying the energy flow coupling relationships to the structures junctions. Park et al. [8,9] developed the energy flow models

\footnotetext{
*Corresponding author. Tel.: +822880 8757; Fax: +82 2888 9298; E-mail: syh@snu.ac.kr.
} 


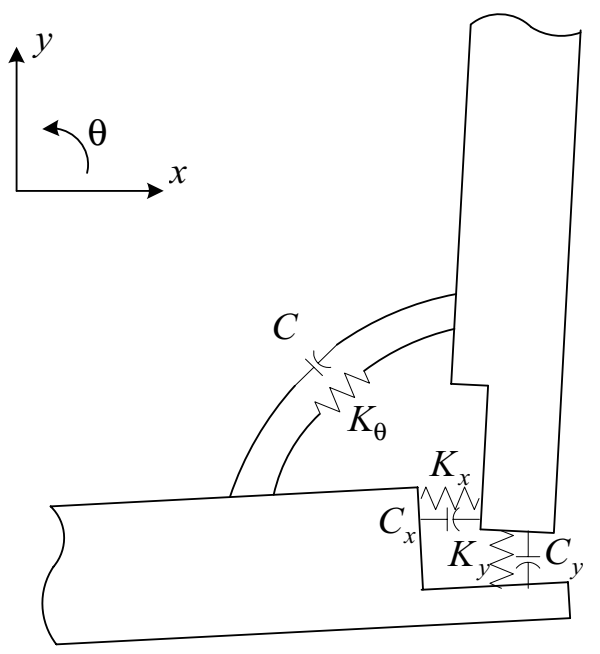

Fig. 1. The joint model.

of in-plane waves in isotropic thin plates and flexural waves in orthotropic thin plates. Seo et al. [10] extended this method to energy flows in reinforced beam-plate coupled structures.

Up to now, most EFAs have been developed and applied to model the dynamics of assembled structures, such as rods, beams, membranes and plates, based on the assumption of rigid joints. The joints in practical structures, however, are often compliant, and this compliance may greatly affect the behaviour of an assembled structure and the transmission of vibrational energy through its components.

The aim of this work is to develop adequate non-conservative joint modeling technique that can be used to predict the vibrational energy and intensity distribution of beam structures vibrating in medium-to-high frequency ranges. Numerical analyses are performed for finite coupled structures connected at a certain angle and excited by a transverse harmonic forces. The effect of change in the joint properties, the frequency, the coupling angle, and the internal loss factor on the vibrational energy and intensity distribution were investigated.

\section{Wave transmission analysis of beam networks with compliant and dissipative joints}

Waves propagating along one component will eventually encounter a joint. A part of the incident wave will be reflected at each joint, while some of the incident wave will be transmitted through the joint. The attenuation of the incident waves transmitted through a joint is largely governed by the impedance and the orientation of the joint. Cremer et al. [11] have studied the transmission of waves through rigid joints of different configurations to find wave transmission coefficients. Horner and White [12] have used the same approach of Cremer et al. to find the wave transmission coefficients of vibrational power transmitted through rigid bends in built-up structures. However, a structure with a rigid joint is not always practical. In general, a linear spring-dashpot model is necessary to model the joint adequately. In this section, the attenuation of the incident waves under the influence of a joint with compliance is predicted.

Riveted, bolted and welded joints can dissipate more energy than the material internal loss factor [13]. Thus, a spring-dashpot model can be appropriate to characterize a joint. Yoshimura and Okushima [14,15] identified the stiffness and the damping coefficients successfully using linear spring-dashpot models. The similar linear springdashpot models are adopted in this work. There are three degrees of freedom at a certain angle joint, $x$ and $y$ directional displacement and rotation, and thus three sets of spring-dashpot models are required as shown in Fig. 1.

Figure 2 shows two semi-infinite beams of different material and/or geometrical properties joined at $x_{1}=x_{2}=0$. Either a flexural or longitudinal wave can be incident upon the joint from the incident beam 1 (beam 2). When a flexural wave of amplitude $\overline{A_{f 1}}$ or a longitudinal wave of amplitude $\overline{P_{l 1}}$ is incident upon the joint, both transmitted and reflected flexural and longitudinal waves are generated in each of the semi-infinite beams. The transverse and 


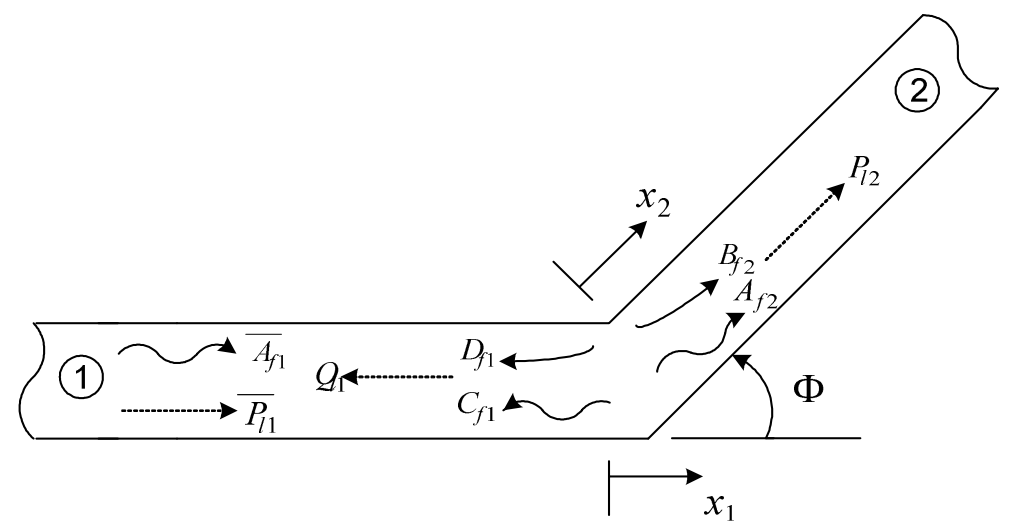

Fig. 2. Transmitted and reflected flexural and longitudinal waves due to the incident flexural or longitudinal wave.

longitudinal displacements $\left(w_{1}\right.$ and $\left.u_{1}\right)$ of incident beam 1 can be represented as follows:

$$
w_{1}\left(x_{1}\right)=\overline{A_{f 1}} e^{-j k_{f 1} x_{1}}+C_{f 1} e^{j k_{f 1} x_{1}}+D_{f 1} e^{k_{f 1} x_{1}}
$$

and

$$
u_{1}\left(x_{1}\right)=\overline{P_{l 1}} e^{-j k_{l 1} x_{1}}+Q_{l 1} e^{k_{l 1} x_{1}}
$$

where $k_{f 1}$ and $k_{l 1}$ are the flexural and longitudinal wavenumbers in the incident beam 1, respectively. In Eq. (1), the first term is the incident flexural propagating wave, the second term is the flexural reflected propagating wave and the third is the reflected decaying nearfield wave. In Eq. (2), the first term is the incident longitudinal propagating wave and the second term is the reflected decaying nearfield wave. The transverse and longitudinal displacements $\left(w_{2}\right.$ and $\left.u_{2}\right)$ in the transmitted beam 2 can be represented as follows:

$$
w_{2}\left(x_{2}\right)=A_{f 2} e^{-j k_{f 2} x_{2}}+B_{f 2} e^{-k_{f 2} x_{2}}
$$

and

$$
u_{2}\left(x_{2}\right)=P_{l 2} e^{-j k_{l 2} x_{2}},
$$

where $k_{f 2}$ and $k_{l 2}$ are the flexural and longitudinal wavenumbers in the transmitted beam 2, respectively. In Eq. (3), the first term is the flexural transmitted propagating wave and the second term is the transmitted decaying nearfield wave. In Eq. (4), the term is the transmitted longitudinal propagating wave.

At the joint, six equilibrium conditions must be satisfied. Three equilibrium conditions impose the equilibrium of forces and moment about the joint. The expressions for the axial force, shear force and moment $(F, V$ and $M)$, respectively, are

$$
\begin{aligned}
& F=E A \frac{d u}{d x}, \\
& V=-E I \frac{d^{3} w}{d x^{3}}
\end{aligned}
$$

and

$$
M=E I \frac{d^{2} w}{d x^{2}},
$$

where $E$ is the Young's modulus, $A$ is the cross-sectional area and $I$ is the area moment of inertia of the beam. The joint force in the horizontal direction is summed to zero as follows:

$$
-F_{1}+F_{2} \cos \Phi-V_{2} \sin \Phi=0
$$

where $F_{1}$ and $F_{2}$ are the axial forces in beams 1 and 2, respectively, and $V_{2}$ is the shear force in beam 2 . The joint force in the vertical direction is summed to zero as follows: 


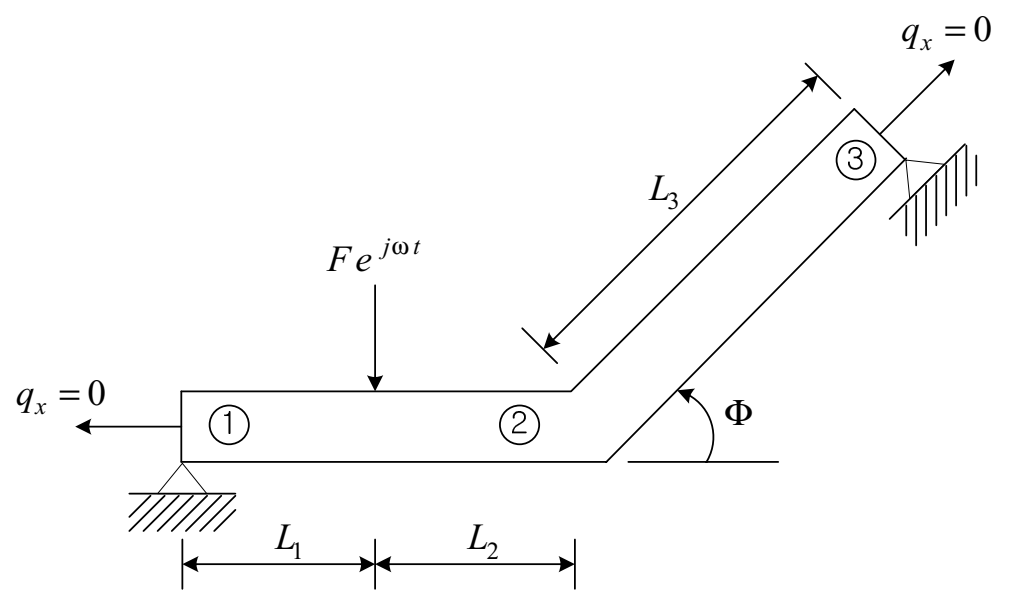

Fig. 3. Two-beam structure coupled with a certain angle $\Phi$ and zero intensity boundary conditions.

$$
-V_{1}+V_{2} \cos \Phi+F_{2} \sin \Phi=0
$$

where $V_{1}$ and $V_{2}$ are the shear forces in beams 1 and 2, respectively, and $F_{2}$ is the axial force in beam 2. The moment about the joint is summed to zero as follows:

$$
M_{2}-M_{1}=0,
$$

where $M_{1}$ and $M_{2}$ are the moments in beams 1 and 2, respectively. The other three equilibrium conditions impose compatibility of displacement and slope. The joint between the two beams is assumed to have the characteristics of three linear spring-dashpot systems. The impedances in the $\mathrm{x}$-direction, the $\mathrm{y}$-direction and the rotation direction $\left(Z_{x}, Z_{y}\right.$ and $\left.Z_{\theta}\right)$ are represented as follows:

$$
\begin{aligned}
& Z_{x}=\left(\frac{K_{x}}{j \omega}\right)+C_{x}, \\
& Z_{y}=\left(\frac{K_{y}}{j \omega}\right)+C_{y}
\end{aligned}
$$

and

$$
Z_{\theta}=\left(\frac{K_{\theta}}{j \omega}\right)+C_{\theta} .
$$

where $K_{x}, K_{y}$ and $K_{\theta}$ are the spring strengths in the x-direction, the y-direction and the rotation direction, respectively, and $C_{x}, C_{y}$ and $C_{\theta}$ are the damper strengths in the x-direction, the y-direction and the rotation direction, respectively. The compatibility of axial displacement and transverse displacement, respectively, are

$$
-F_{2} \cos \Phi+V_{2} \sin \Phi-Z_{x}\left(u_{1}+w_{2} \sin \Phi-u_{2} \cos \Phi\right)=0
$$

and

$$
-V_{2} \cos \Phi-F_{2} \sin \Phi-Z_{y}\left(w_{1}-w_{2} \cos \Phi-u_{2} \sin \Phi\right)=0 .
$$

Also, the compatibility of slope leads to the following relation:

$$
M_{2}-Z_{\theta}\left(\frac{d w_{1}}{d x_{1}}-\frac{d w_{2}}{d x_{2}}\right)=0 .
$$

When all six conditions at the joint are applied, the unknown complex amplitudes, $C_{f 1}, D_{f 1}, Q_{l 1}, A_{f 2}, B_{f 2}$ and $P_{l 2}$ are obtained numerically by the given material and geometrical properties of the beams. Subsequently, 

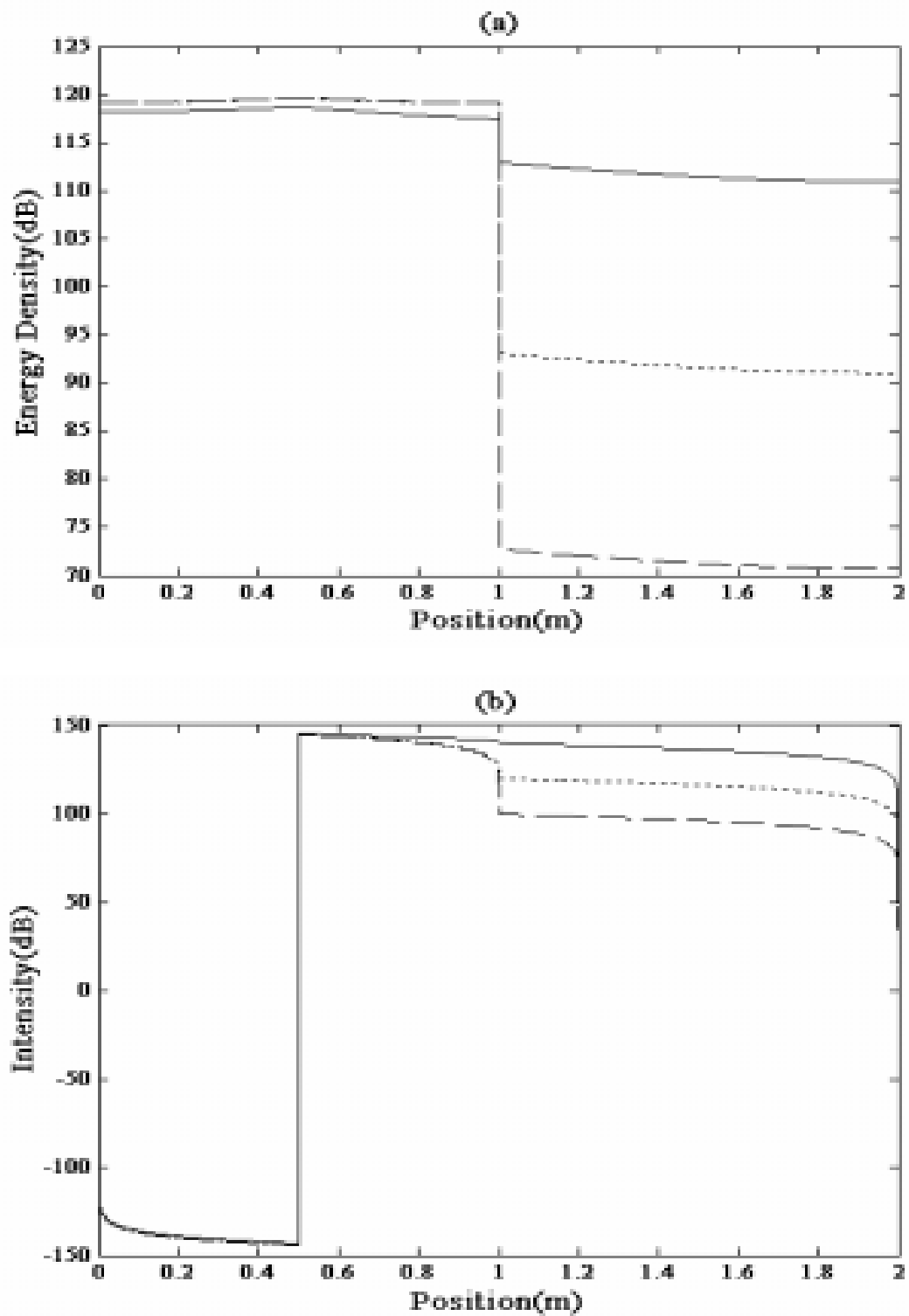

Fig. 4. Energy and intensity distributions of two-beam structure coupled with $\Phi=90^{\circ}$ when $f=1 \mathrm{kHz}$ and $\eta=0.1$. (a) flexural wave energy, (b) flexural wave intensity.

the flexural and longitudinal power transmission and reflection coefficients can be computed from the complex amplitudes.

Before flexural and longitudinal power transmission and reflection coefficients can be derived, an expression for the time-averaged power in a beam must be obtained. For a harmonic excitation, the time-average power in a beam is

$$
<P>_{f}=\frac{1}{2} R e\left\{-V \frac{d w^{*}}{d t}-M \frac{d^{2} w^{*}}{d x d t}\right\}
$$

and

$$
<P>{ }_{l}=\frac{1}{2} \operatorname{Re}\left\{-F \frac{d u^{*}}{d t}\right\},
$$



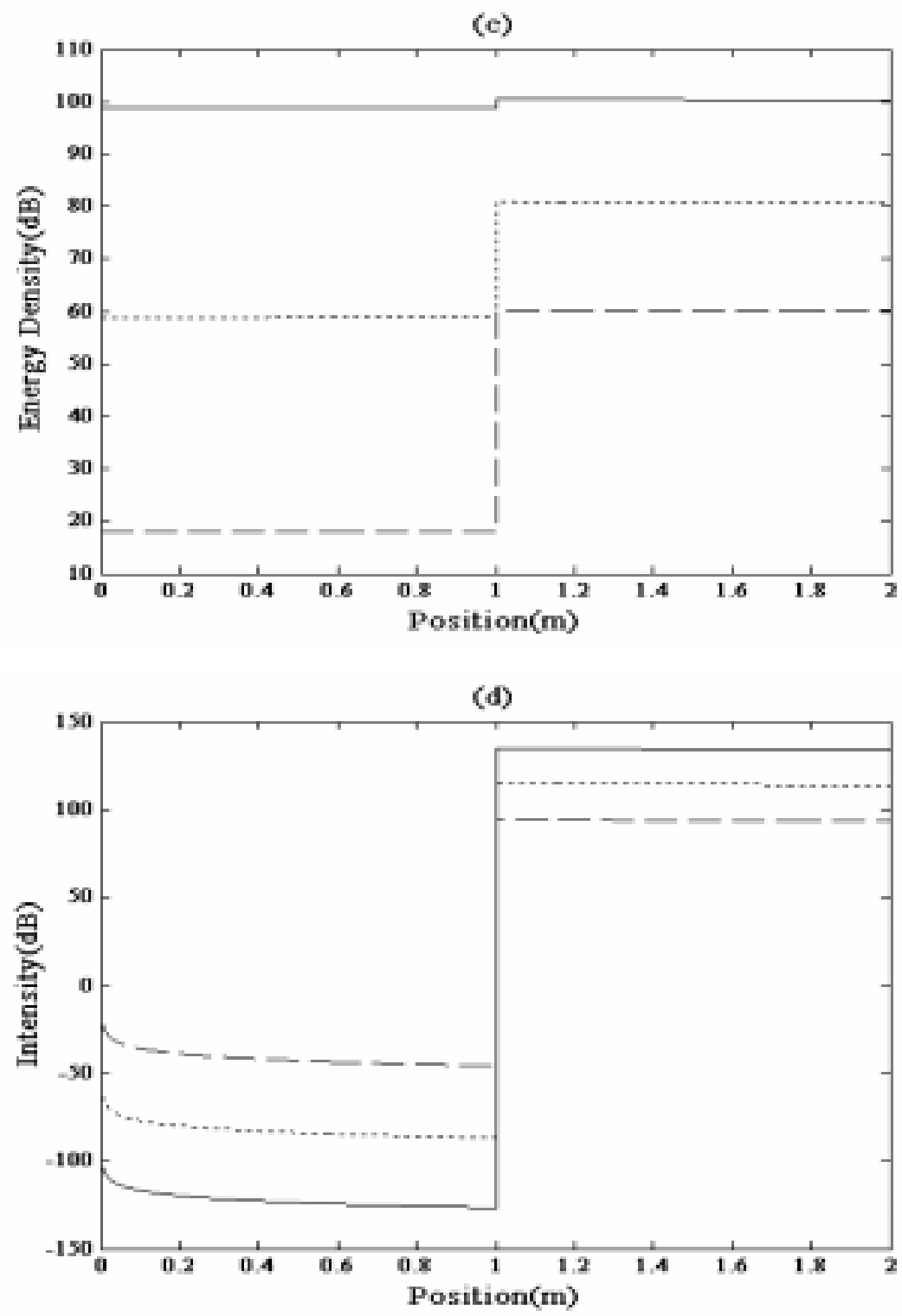

Fig. 4, continued. (c) longitudinal wave energy, (d) longitudinal wave intensity. _ rigid joint; ....... $K_{x}=K_{y}=10^{9} \mathrm{~N} / \mathrm{m}, K_{\theta}=10^{6} \mathrm{Nm} / \mathrm{rad}$, $C_{x}=C_{y}=10^{7} \mathrm{Ns} / \mathrm{m}, C_{\theta}=10^{4} \mathrm{Nms} / \mathrm{rad} ;-\cdots K_{x}=K_{y}=10^{8} \mathrm{~N} / \mathrm{m}, K_{\theta}=10^{5} \mathrm{Nm} / \mathrm{rad}, C_{x}=C_{y}=10^{6} \mathrm{Ns} / \mathrm{m}, C_{\theta}=10^{3} \mathrm{Nms} / \mathrm{rad}$.

where $d w^{*} / d t$ is the complex conjugate of the transverse velocity, $d^{2} w^{*} / d x d t$ is the complex conjugate of the angular velocity and $u^{*}$ is the complex conjugate of the longitudinal velocity. For an incident flexural and longitudinal wave of amplitudes, $\overline{A_{f 1}}$ and $\overline{P_{l 1}}$, the time-averaged power is written as

$$
<P>_{f}=E I k_{f}^{3} w\left|\overline{A_{f 1}}\right|^{2}
$$

and

$$
<P>_{l}=\frac{1}{2} E A k_{l} w\left|\overline{P_{l 1}}\right|^{2} .
$$

The balance of energy applied to the non-conservative joint of two semi-infinite beams is expressed as

$$
<P>_{\alpha 1 \text { inc }}=<P>_{\text {f1ref }}+<P>_{\text {l1ref }}+<P>_{\text {f2trans }}+<P>_{\text {l2trans }}+<P>_{\text {diss }},
$$



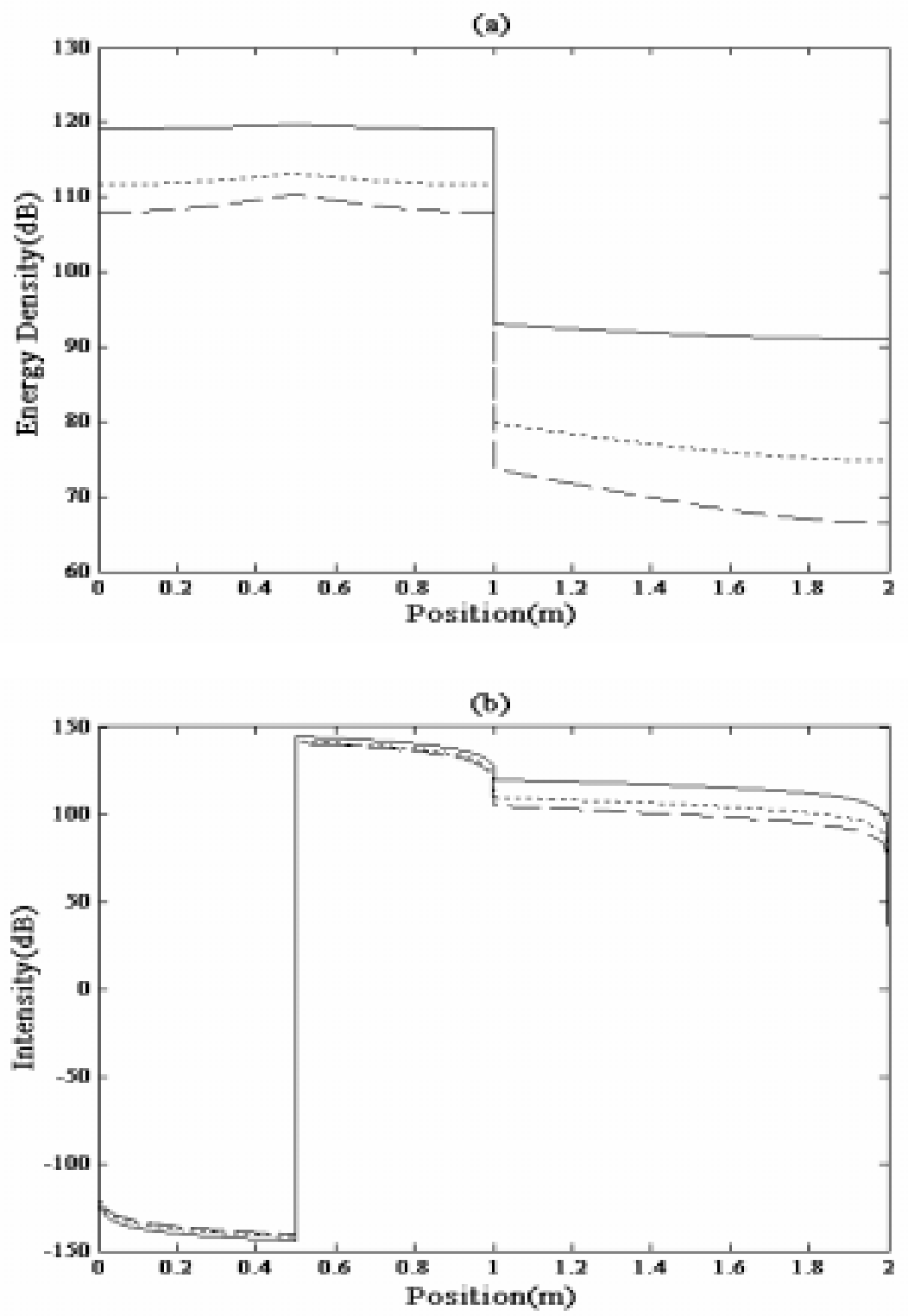

Fig. 5. Energy and intensity distributions of two-beam structure coupled with $\Phi=90^{\circ}$ when $K_{x}=K_{y}=10^{9} \mathrm{~N} / \mathrm{m}, K_{\theta}=10^{6} \mathrm{Nm} / \mathrm{rad}, C_{\theta}=$ $10^{4} \mathrm{Nms} / \mathrm{rad}, C_{x}=C_{y}=10^{7} \mathrm{Ns} / \mathrm{m}$ and $\eta=0.1$. (a) flexural wave energy, (b) flexural wave intensity.

where the subscript $\alpha$ indicates a flexural wave $(f)$ or a longitudinal wave $(l)$. The incident power is the sum of the reflected, transmitted and dissipated power. When both sides of Eq. (21) are divided by $\langle P\rangle\rangle_{\alpha 1 \text { inc }}$, the following equation can be obtained:

$$
\begin{aligned}
\frac{\langle P\rangle_{\alpha 1 \text { inc }}}{\langle P\rangle_{\alpha 1 \text { inc }}} & =\frac{\left\langle P>_{f 1 \text { ref }}\right.}{\left\langle P>_{\alpha 1 \text { inc }}\right.}+\frac{\left\langle P>_{\text {l1ref }}\right.}{\left\langle P>_{\alpha 1 \text { inc }}\right.}+\frac{\left\langle P>_{f 2 \text { trans }}\right.}{\left\langle P>_{\alpha 1 \text { inc }}\right.}+\frac{\left\langle P>_{\text {l2trans }}\right.}{\left\langle P>_{\alpha 1 \text { inc }}\right.}+\frac{\left\langle P>_{\text {diss }}\right.}{\left\langle P>_{\alpha 1 \text { inc }}\right.} \\
& =\gamma_{\alpha f 11}+\gamma_{\alpha l 11}+\tau_{\alpha f 12}+\tau_{\alpha l 12}+\zeta=1 .
\end{aligned}
$$

The flexural and longitudinal reflection coefficients $\left(\gamma_{\alpha f 11}\right.$ and $\left.\gamma_{\alpha l 11}\right)$ are the ratios of the reflected to the incident power, respectively, and the flexural and longitudinal transmission coefficients $\left(\tau_{\alpha f 12}\right.$ and $\left.\tau_{\alpha l 12}\right)$ are the ratios of 

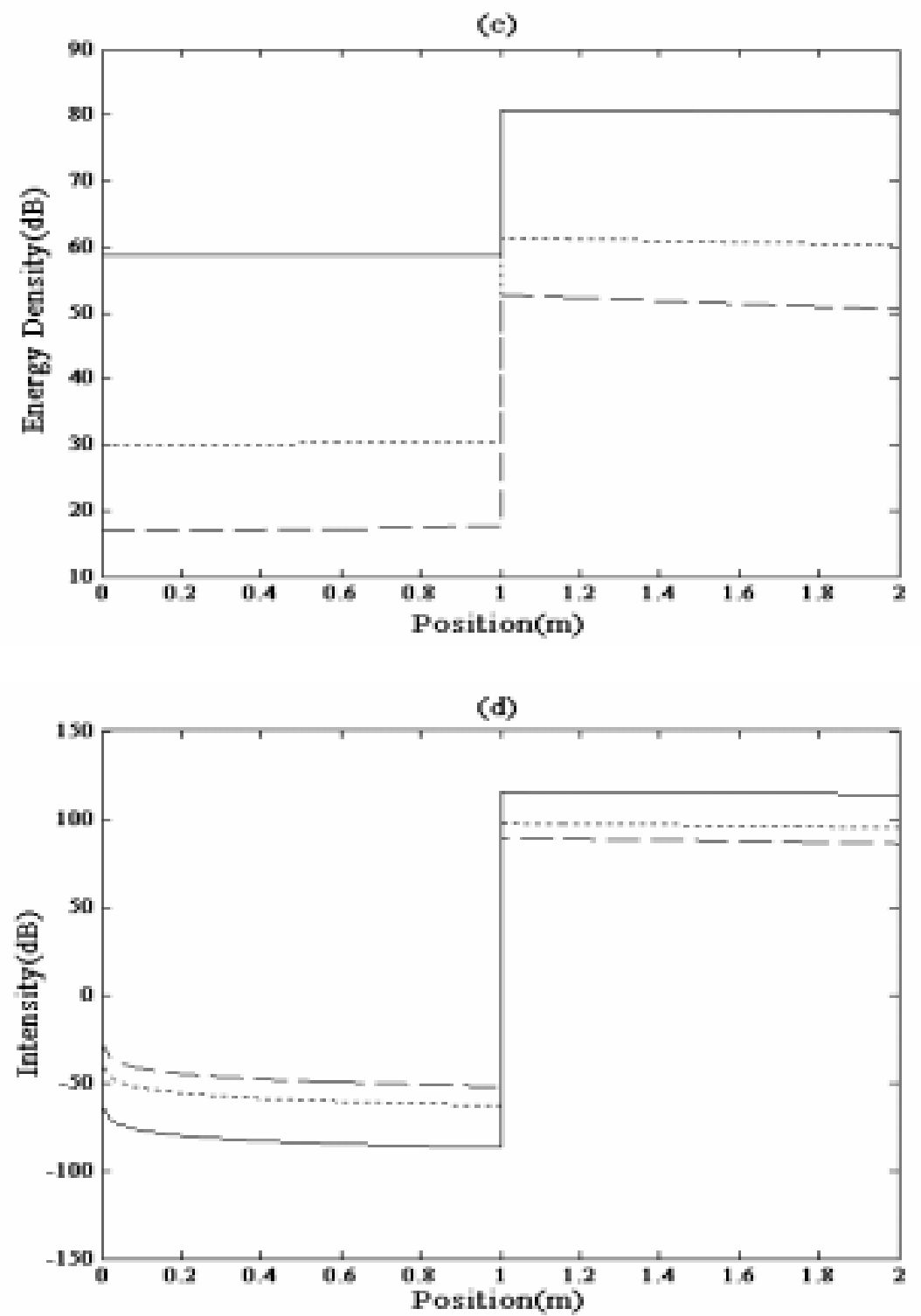

Fig. 5, continued. (c) longitudinal wave energy, (d) longitudinal wave intensity. $f=1 \mathrm{kHz} ; \ldots \ldots . f=3 \mathrm{kHz} ;-.-.-f=5 \mathrm{kHz}$.

the transmitted to the incident power, respectively. The dissipation coefficient $(\zeta)$ is the ratio of the dissipated to the incident power.

\section{Energy flow analysis in beam networks}

In this section, the power transmission and reflection coefficients at the non-conservative joint of two semi-infinite beams, Eq. (22), and the equation of the flexural and longitudinal waves developed by Bernhard $[3,4]$ are applied to the coupled beam structure. The coupled structure is composed of two beams connected at a certain angle and excited by a transverse harmonic point force. The power due to the exciting force is applied to beam 1 as shown in Fig. 3, and zero intensity boundary conditions for each wave component at the beam edges are applied. 

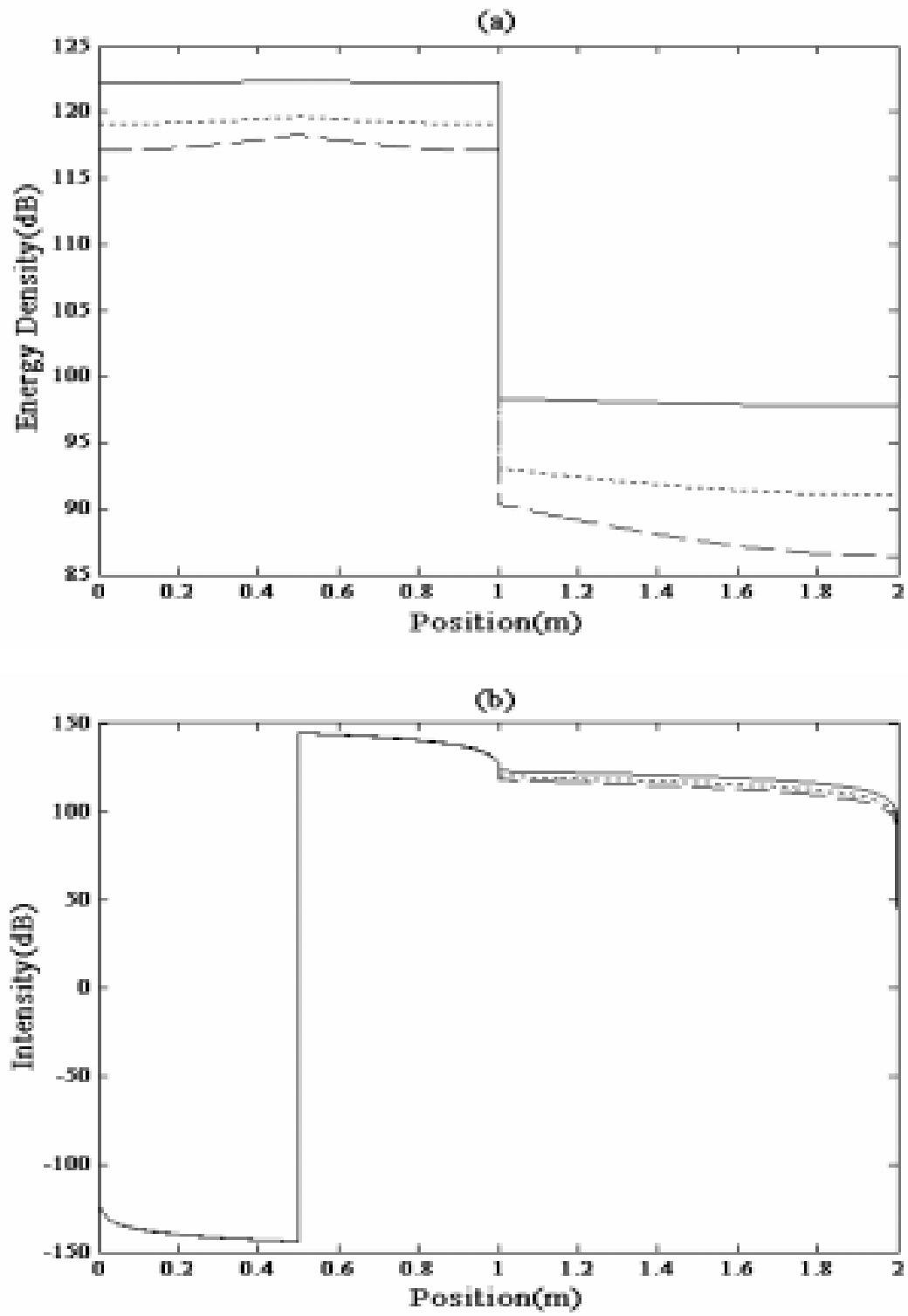

Fig. 6. Energy and intensity distributions of two-beam structure coupled with $\Phi=90^{\circ}$ when $K_{x}=K_{y}=10^{9} \mathrm{~N} / \mathrm{m}, K_{\theta}=10^{6} \mathrm{Nm} / \mathrm{rad}, C_{\theta}=$ $10^{4} \mathrm{Nms} / \mathrm{rad}, C_{x}=C_{y}=10^{7} \mathrm{Ns} / \mathrm{m}$ and $f=1 \mathrm{kHz}$. (a) flexural wave energy, (b) flexural wave intensity.

The energy density of each wave component, $\left\langle\overline{e_{\alpha}}>_{i}\right.$, can be represented as a sum of positively and negatively moving energy densities as:

$$
<\overline{e_{\alpha}}>_{\beta}=<\overline{e_{\alpha}}>_{\beta}^{+}+<\overline{e_{\alpha}}>_{\beta}^{-},
$$

where

$$
<\overline{e_{\alpha}}>_{\beta}^{+}=A_{\alpha \beta}^{+} \exp \left(-\frac{\eta \omega}{c_{g \alpha \beta}} x\right)
$$

and

$$
<\overline{e_{\alpha}}>_{\beta}^{-}=A_{\alpha \beta}^{-} \exp \left(\frac{\eta \omega}{c_{g \alpha \beta}} x\right) .
$$



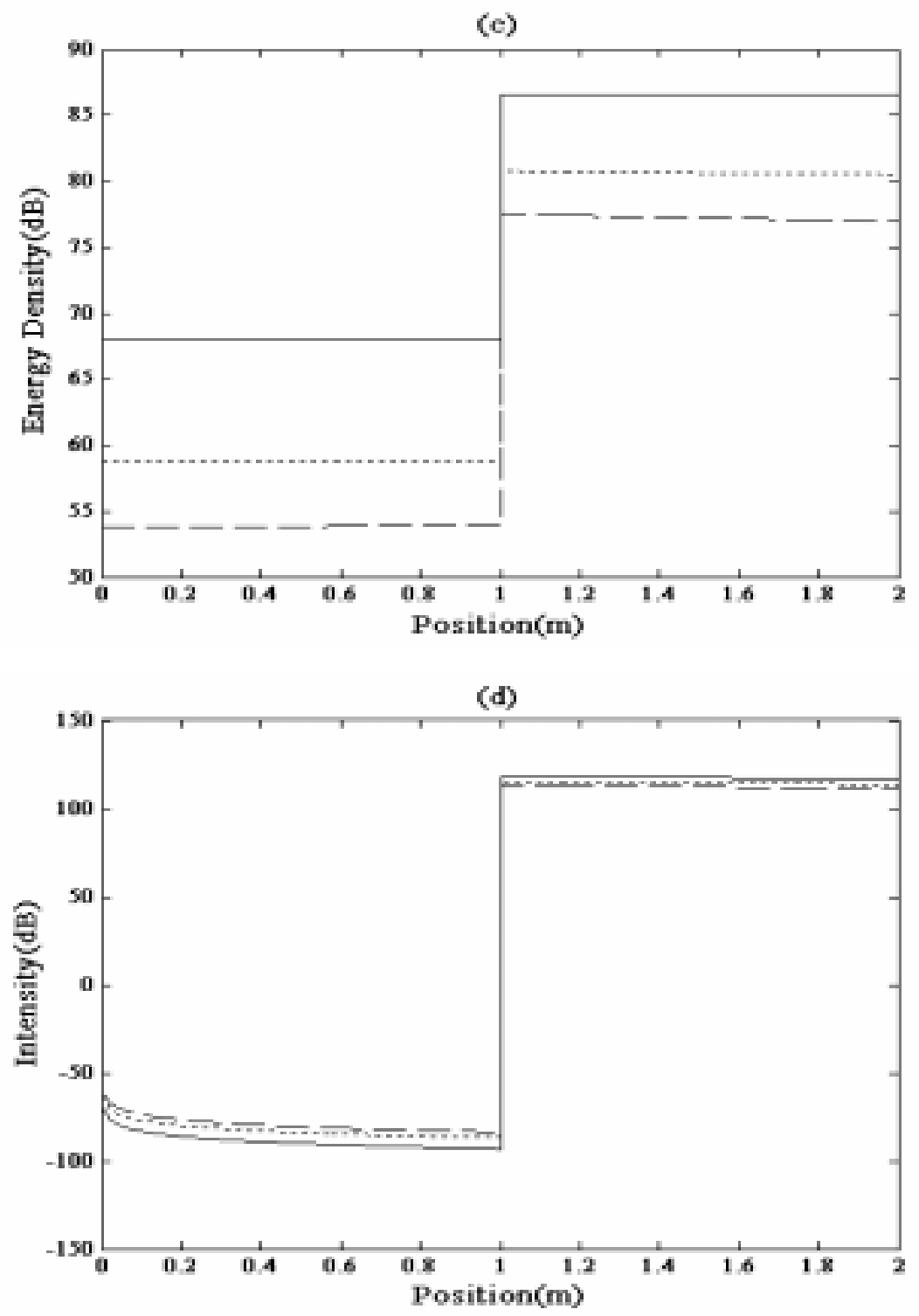

Fig. 6, continued. (c) longitudinal wave energy, (d) longitudinal wave intensity. $\eta=0.05$ $\eta=0.1 ;-\cdots-\eta .15$

The subscript $\alpha$ indicates a flexural wave $(f)$ or a longitudinal wave $(l)$. The subscript $\beta$ indicates the region (1), (2) or (3), demarcated by the position of the input power and the joint of the two beams as illustrated in Fig. 3 . The superscripts $(+)$ and $(-)$ represent wave propagation in the $+x$ and $-x$ direction, respectively.

The intensity for any wave types, $\left\langle\overline{q_{\alpha}}>_{i}\right.$, can be represented as a difference of positively and negatively moving intensity as:

$$
<\overline{q_{\alpha}}>_{\beta}=<\overline{q_{\alpha}}>_{\beta}^{+}-<\overline{q_{\alpha}}>_{\beta}^{-},
$$

where

$$
<\overline{q_{\alpha}}>_{\beta}^{+}=c_{g \alpha \beta} e_{\alpha \beta}^{+}
$$

and

$$
<\overline{q_{\alpha}}>_{\beta}^{-}=c_{g \alpha \beta} e_{\alpha \beta}^{-} .
$$



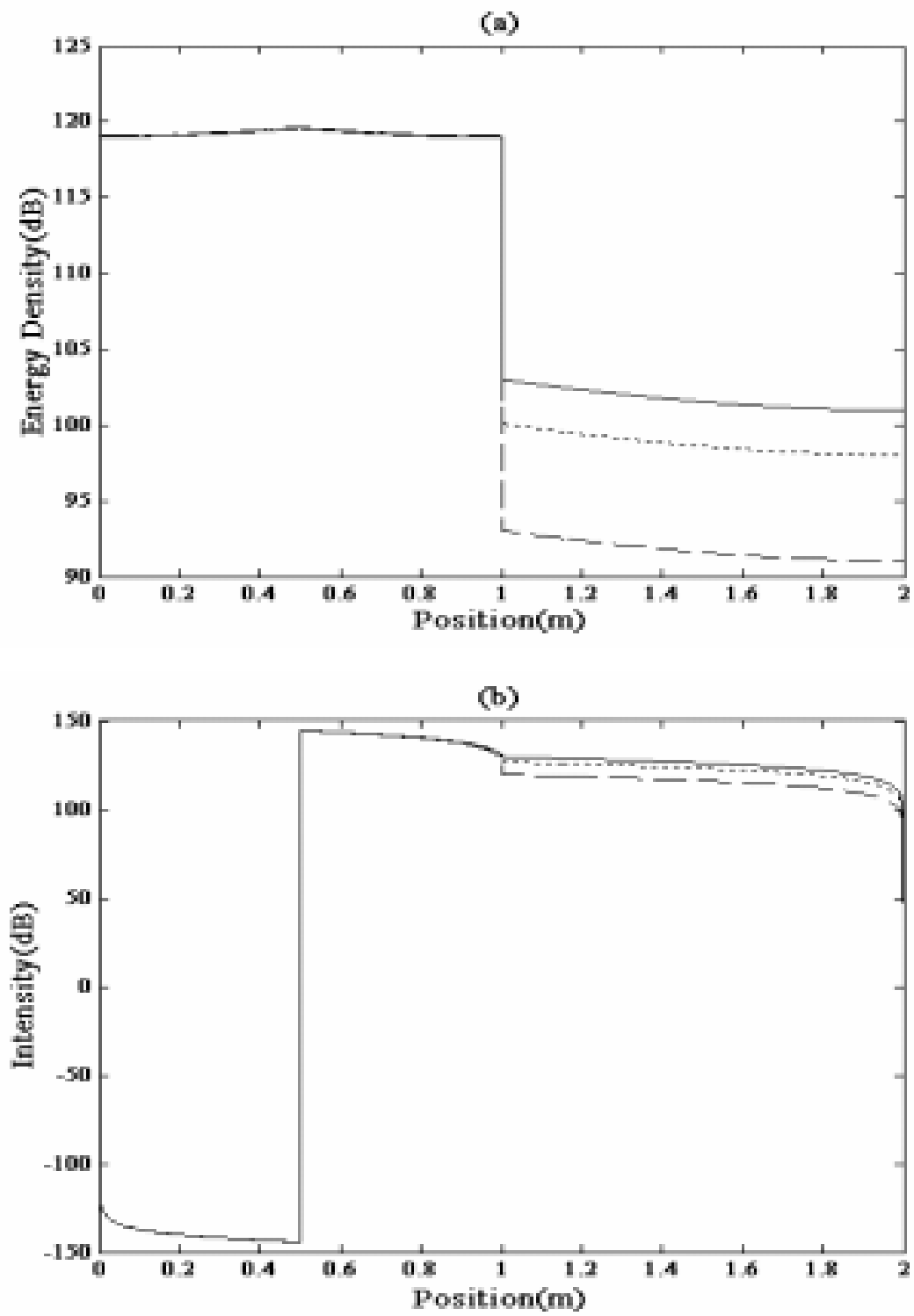

Fig. 7. Energy and intensity distributions of two-beam structure coupled when $f=1 \mathrm{kHz}, K_{x}=K_{y}=10^{9} \mathrm{~N} / \mathrm{m}, K_{\theta}=10^{6} \mathrm{Nm} / \mathrm{rad}$, $C_{x}=C_{y}=10^{7} \mathrm{Ns} / \mathrm{m}, C_{\theta}=10^{4} \mathrm{Nms} / \mathrm{rad}$ and $\eta=0.1$. (a) flexural wave energy, (b) flexural wave intensity.

To determine the unknowns $A_{\alpha \beta}^{+}$and $A_{\alpha \beta}^{-}$in Eqs (24) and (25), there are twelve boundary conditions that must be enforced. Four boundary conditions impose at the end of beam. Since no other structural elements are attached to the ends of the beam, the energy can not flow out of the ends of the beam. All the energy incident upon these boundaries will be reflected. Hence, we write the intensity boundary conditions as follows:

$$
<\overline{q_{\alpha}}>_{1}^{+}=<\overline{q_{\alpha}}>_{1}^{-}
$$

and

$$
<\overline{q_{\alpha}}>_{3}^{+}=<\overline{q_{\alpha}}>_{3}^{-} .
$$

The other four boundary conditions impose at the excitation point. The input power due to a point exciting force $Q$ is defined as: 

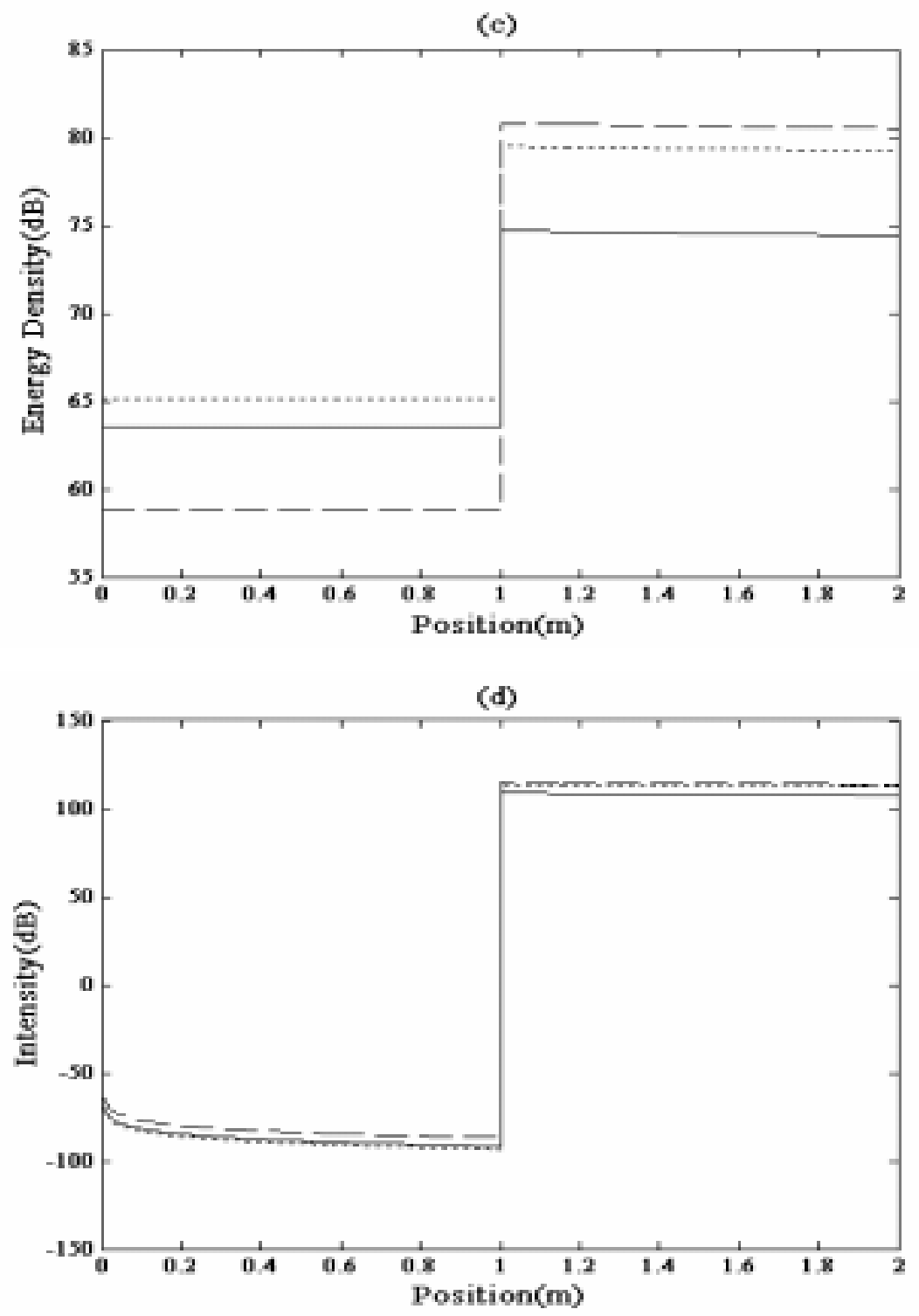

Fig. 7, continued. (c) longitudinal wave energy, (d) longitudinal wave intensity. $\Phi=30^{\circ}$ $\Phi=60^{\circ} ;-\cdots \Phi=90^{\circ}$.

$$
Q=\frac{1}{2}\left|F^{2}\right| \operatorname{Re}\left(\frac{1}{Z}\right),
$$

where $Z$ is the impedance of infinite beam and given by

$$
Z=2 \rho A c_{f}(1+j) .
$$

Here, $\rho, A$ and $c_{f}$ are the material density, the cross-sectional area and the phase speed of flexural waves, respectively. From the equilibriums of intensity and the continuity of energy at the excitation point, the following relations are satisfied as:

$$
\begin{aligned}
& <\overline{e_{\alpha}}>_{1}^{+}+<\overline{e_{\alpha}}>_{1}^{-}=<\overline{e_{\alpha}}>_{2}^{+}+<\overline{e_{\alpha}}>_{2}^{-}, \\
& \left(<\overline{q_{f}}>_{2}^{+}-<\overline{q_{f}}>_{2}^{-}\right)-\left(<\overline{q_{f}}>_{1}^{+}-<\overline{q_{f}}>_{1}^{-}\right)=Q,
\end{aligned}
$$




$$
\left(<\overline{q_{l}}>_{2}^{+}-<\overline{q_{l}}>_{2}^{-}\right)-\left(<\overline{q_{l}}>_{1}^{+}-<\overline{q_{l}}>_{1}^{-}\right)=0 \text {. }
$$

The last four boundary conditions impose at the joint of beam 1 and 2, where the incident waves are converted to other types of waves, and the power transmission and reflection of the incident wave must be examined. From the previous section, the power flowing away from the non-conservative joint of two semi-infinite beams are expressed as follows:

$$
\begin{aligned}
& <\overline{q_{f}}>_{2}^{-}=\gamma_{f f 22}<\overline{q_{f}}>_{2}^{+}+\gamma_{l f 22}<\overline{q_{l}}>_{2}^{+}+\tau_{f f 32}<\overline{q_{f}}>_{3}^{-}+\tau_{l f 32}<\overline{q_{l}}>_{3}^{-}, \\
& <\overline{q_{l}}>_{2}^{-}=\gamma_{f l 22}<\overline{q_{f}}>_{2}^{+}+\gamma_{l l 22}<\overline{q_{l}}>_{2}^{+}+\tau_{f l 32}<\overline{q_{f}}>_{3}^{-}+\tau_{l l 32}<\overline{q_{l}}>_{3}^{-}, \\
& <\overline{q_{f}}>_{3}^{+}=\tau_{f f 23}<\overline{q_{f}}>_{2}^{+}+\tau_{l f 23}<\overline{q_{l}}>_{2}^{+}+\gamma_{f f 33}<\overline{q_{f}}>_{3}^{-}+\gamma_{l f 33}<\overline{q_{l}}>_{3}^{-}, \\
& <\overline{q_{f}}>_{3}^{+}=\tau_{f l 23}<\overline{q_{f}}>_{2}^{+}+\tau_{l l 23}<\overline{q_{l}}>_{2}^{+}+\gamma_{f l 33}<\overline{q_{f}}>_{3}^{-}+\gamma_{l l 33}<\overline{q_{l}}>_{3}^{-} .
\end{aligned}
$$

where $\tau_{m n i j}$ is the $n$ wave type power transmission coefficient in region $j$ due to the incident $m$ wave type in region $i$ ( $i, j=(2)$, (3); $m, n=f, l$ ), and $\gamma_{m n i j}$ is the $n$ wave type power reflection coefficient in region $i$ due to the incident $m$ wave type in region $i$. From the boundary conditions, the unknowns in each wave of the amplitude are determined, and the energy and intensity for all the wave types are finally obtained.

\section{Numerical examples}

In this section, several numerical analyses are performed for the finite coupled structures connected at a certain angle. The derived non-conservative joints and developed wave energy equation were applied. The joint properties, the frequency, the coupling angle and the internal loss factor were changed to evaluate the proposed methods in predicting medium-to-high frequency vibrational energy and intensity distributions. The decibel scales of the energy density and intensity referenced to $10^{-12} \mathrm{~J} / \mathrm{m}^{2}$ and $10^{-12} \mathrm{~W} / \mathrm{m}^{2}$ are used in all figures of the example results. Both beams 1 and 2 shown in Fig. 3 are $1 \mathrm{~m} \times 10 \mathrm{~mm}$ in dimensions and the material properties of the beam are assumed to be those of steel. The input power is located at $x_{0}=0.5 \mathrm{~m}$ in beam 1 and its amplitude is $F=10000 \mathrm{~N}$. The internal loss factors of two wave types are assumed to have the same value of $\eta$.

Figure 4(a)-(d) show that when the joint property changes, the developed non-conservative joint successfully predicts the joint character of practical structures vibrating in the medium-to-high frequency ranges. On the whole, both transmitted flexural and longitudinal energy levels can be controlled to some extent by varying either the stiffness or the damper of the joint. The flexural wave energy is discontinuous at the beam joint showing the lower level in beam 2 as seen in Fig. 4(a), and the energy level of longitudinal waves in beam 2 is higher than in beam 1 as shown in Fig. 4(c), as expected. The energy levels of all wave types are nearly constant in each beam. The energy flow pattern of the non-conservative joint is visualized in the intensity distributions of Fig. 4(b) and 4(d). A negative value of intensity indicates that the energy is flowing in the negative direction; a positive value of intensity indicates that the energy is flowing in the positive direction. The excitation source is located at the point of discontinuity in the intensity distribution where the positive and negative energy flows begin. For reference [14], the stiffness of a bolted joint for the size of the chosen steel beam will be of the order of $10^{8}$ to $10^{11} \mathrm{~N} / \mathrm{m}$ depending on the contact pressure of the bolted joint. Also, a bolted joint will have a damping coefficient in the order of about $0^{6} \mathrm{Ns} / \mathrm{m}$ for a unit width and $10 \mathrm{~mm}$ thick steel beam.

In the second example, the importance of the non-conservative joints in longitudinal wave is demonstrated in Fig. 5(a)-(d) for a vibrating in the medium-to-high frequency ranges. As the frequency increases, the global variation of the energy increases and the maximum energy level is reduced. The longitudinal wave energy has maximum value near the location of the input power in the joint. The intensity distribution of Fig. 5(b) and 5(d) shows that the energy transmission paths do not change significantly as the frequency increases. These results verify that the longitudinal wave is important in transmitting the vibration energy of a coupled beam structure at high-frequency ranges.

In the next example, the internal loss factors are changed from $\eta=0.05$ to $\eta=0.15$. The distribution of energy and intensity of two types are shown in Fig. 6(a)-(d). The energy level of a flexural wave dissipates more rapidly than that of a longitudinal wave, which is still nearly constant in each beam, because the group velocity of the 
longitudinal wave is much greater than that of a flexural wave. The energy difference of each wave between beams 1 and 2 at the joint increases as the internal loss factors are increased. The corresponding intensity distributions are illustrated in Fig. 6(b) and 6(d) by hanging the internal loss factor.

In Fig. 7(a)-(d), the distribution of energy and intensity of two types as a dependent variable of the joint angle $\Phi$ vary from 30 to $90^{\circ}$. As $\Phi$ increases, the global variation of the energy increases and reaches maximum variations when the beams are at right angles as expected. The intensity distributions of each wave have nearly constant values as $\Phi$ is increased in Fig. 7(b) and 7(d).

\section{Conclusions}

General expressions for the vibrational energy flows through a plane network of beams are derived based on an energy flow analysis, assuming the joints to be compliant and dissipative, and each joint was modeled by a spring and dashpot. An example of two beams at a certain angle shows that the case of non-conservative joints successfully predict the joint character of practical structures vibrating in the medium-to-high frequency ranges. Moreover, the energy flow pattern of a non-conservative joint is understood. The derived non-conservative joints can be used to evaluate the proposed methods used for predicting medium-to-high frequency vibrational energy and intensity distributions. Further studies on the developments of non-conservative joints in plate network for vibration energy flow analysis are recommended.

\section{Acknowledgements}

This work was supported by the Advanced Ship Engineering Research Center (ASERC) of the Korea Science and Engineering Foundation.

\section{References}

[1] V.D. Belov, S.A. Rybak and B.D. Tartakovskii, Propagation of vibrational energy in absorbing structures, Soviet-Physics Acoustics 23 (1977), 115-119.

[2] D.J. Nefske and S.H. Sung, Power flow finite element analysis of dynamic systems: basic theory and application to beams, Journal of Vibration, Acoustics, Stress and Reliability in Design 111 (1989), 94-100.

[3] J.C. Wohlever and R.J. Bernhard, Mechanical energy flow models of rods and beams, Journal of Sound and Vibration 153 (1992), 1-19.

[4] O.M. Bouthier, R.J. Bernhard and C. Wohlever, Energy and structural intensity formulations of beams and plate vibrations, Proceedings of the 3rd International Congress on Intensity Techniques, Senlis, France, 1990, 37-44.

[5] O.M. Bouthier and R.J. Bernhard, Models of space-averaged energetics of plates, American Institute of Aeronautics and Astronautics Journal 30 (1992), 616-623.

[6] O.M. Bouthier and R.J. Bernhard, Simple models of the energetics of transversely vibrating plates, Journal of Sound and Vibration 182 (1995), 149-164.

[7] P.E. Cho, Energy Flow Analysis of Coupled Structures, Ph.D. Dissertation, Purdue University, 1993.

[8] D.-H. Park, S.-Y. Hong, H.-G. Kil and J.-J. Jeon, Power flow models and analysis of in-plane waves in finite coupled thin plates, Journal of Sound and Vibration 244 (2001), 651-668.

[9] D.-H. Park, S.-Y. Hong and H.-G. Kil, Vibrational energy flow models of finite orthotropic plates, Shock and Vibration 10 (2003), 97-113.

[10] S.-H. Seo, S.-Y. Hong and H.-G. Kil, Power flow analysis of reinforced beam-plate coupled structures, Journal of Sound and Vibration 259 (2003), 1109-1129.

[11] L. Cremer, M. Heckl and E.E. Ungar, Structure-Borne Sound, Springer, Berlin, 1973.

[12] J.L. Horner and R.G. White, Prediction of vibrational power transmission through bends and joints in beam-like structures, Journal of Sound and Vibration 147 (1991), 87-103.

[13] L. Gaul. Wave transmission and energy dissipation at structural and machine joints, Transactions of the American Society of Mechanical Engineers Series L 105 (1983), 409-496.

[14] M. Yoshimura and K. Okushima, Measurement of dynamic rigidity and damping property for simplified joint models and simulation by computer, Annals of the CIRP 25 (1977), 193-198.

[15] M. Yoshimura and K. Okushima, Computer-aided design improvement of machine tool structure incorporating joint dynamics data, Annals of the CIRP 28 (1979), 241-246. 

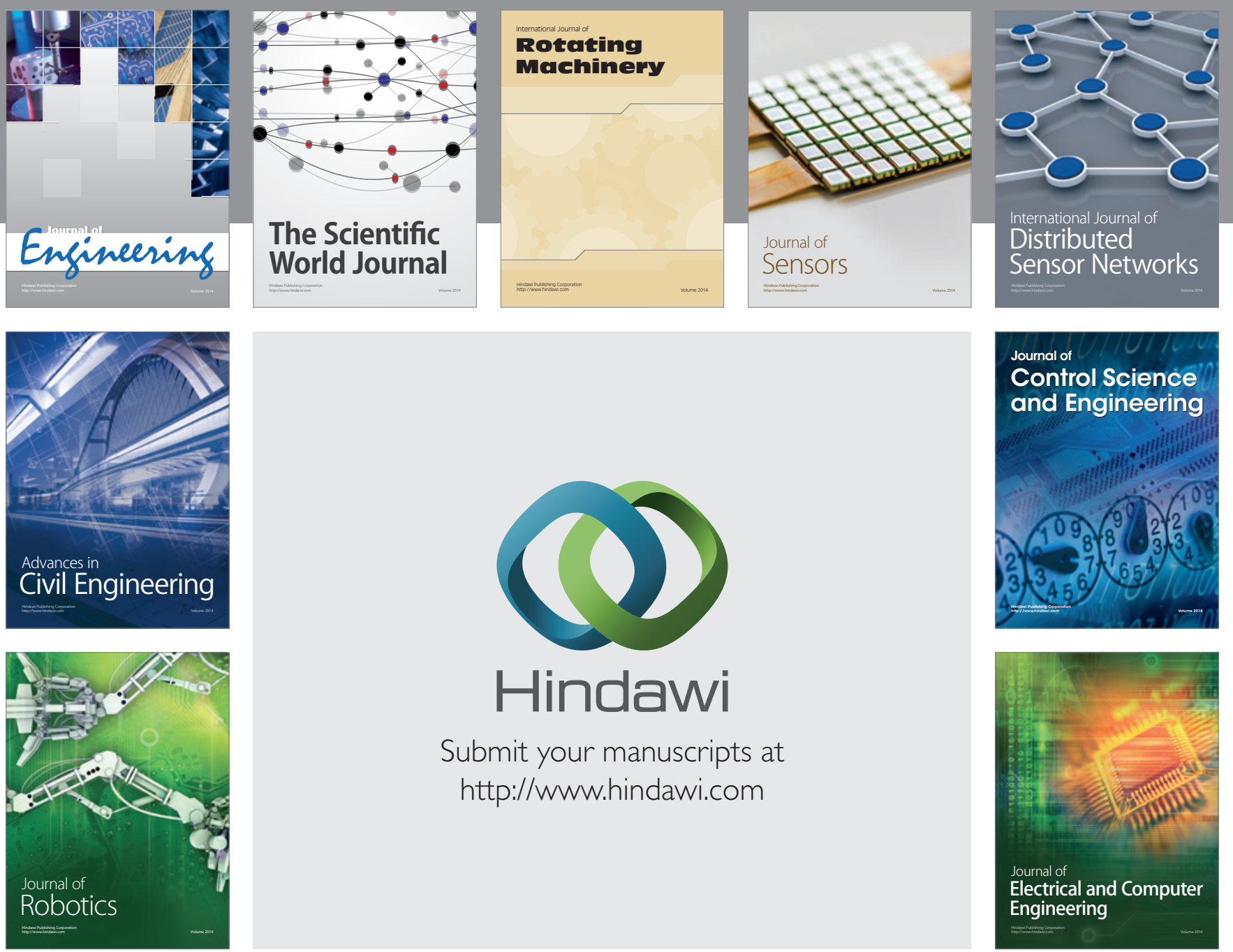

Submit your manuscripts at

http://www.hindawi.com
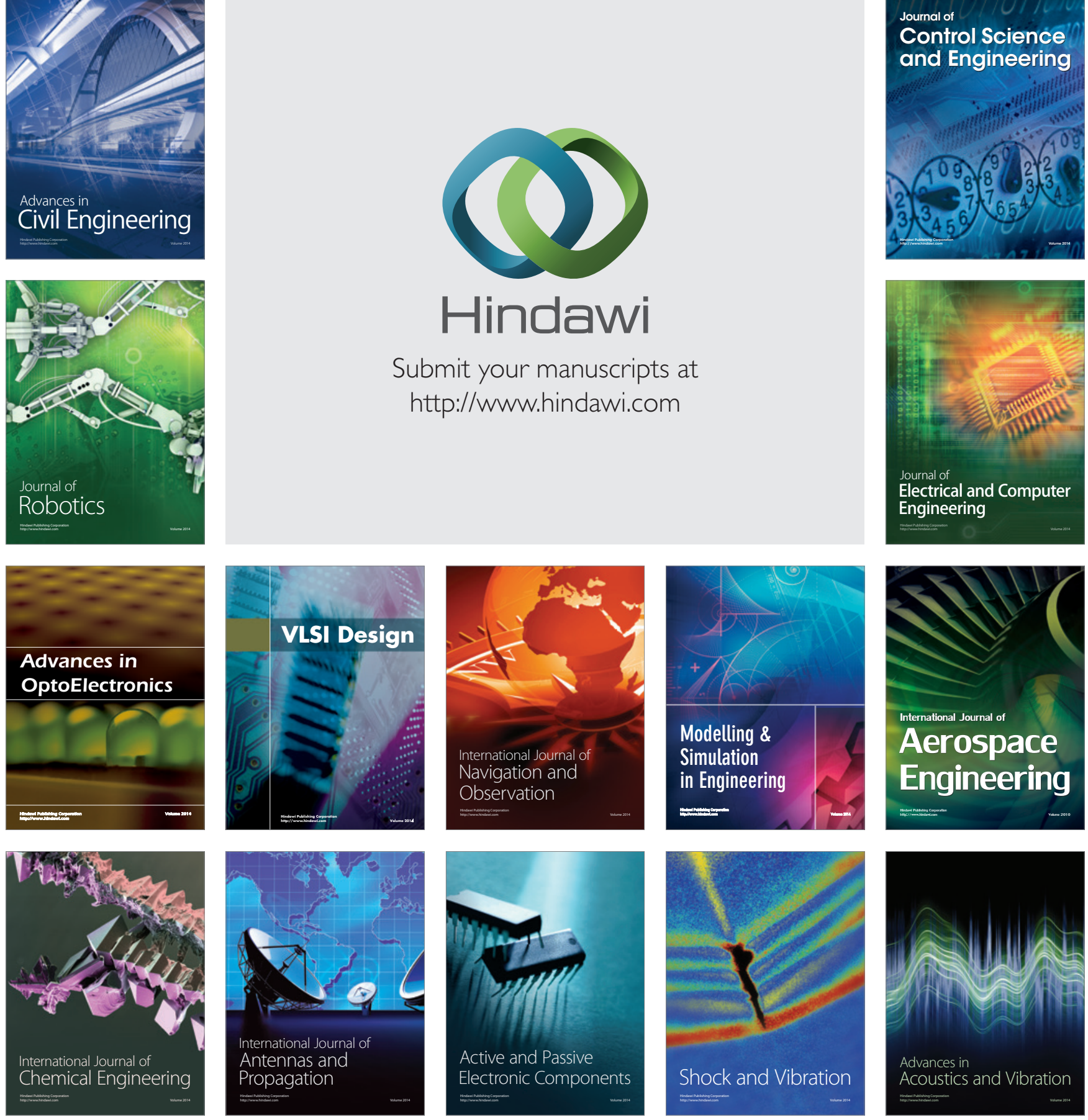